Article

\title{
Effect of Bamboo Flour Grafted Lactide on the Interfacial Compatibility of Polylactic Acid/Bamboo Flour Composites
}

\author{
Xin-Yu Song ${ }^{1}$, Meng Wang ${ }^{1}$, Yun-Xuan Weng ${ }^{1,2, *}$ (D) and Zhi-Gang Huang ${ }^{1,2}$ \\ 1 School of Materials and Mechanical Engineering, Beijing Technology\& Business University, Beijing 100048, \\ China; Songxy722@163.com (X.-Y.S.); dywewm@163.com (M.W.); huangzg@btbu.edu.cn (Z.-G.H.) \\ 2 Beijing Key Laboratory of Quality Evaluation Technology for Hygiene and Safety of Plastics, \\ Beijing Technology and Business University, Beijing 100048, China \\ * Correspondence: wyxuan@th.btbu.edu.cn
}

Received: 21 June 2017; Accepted: 25 July 2017; Published: 30 July 2017

\begin{abstract}
Bamboo flour (BF) was grafted onto lactide (LA) in the molten state using stannous octoate as a catalyst to form BF-g-LA. Then, polylactic acid (PLA) was blended with BF (PLA/BF, 85/15 wt \%) to prepare PLA/BF/BF- $g$-LA composites using BF-g-LA as a compatibilizer. The grafting rate of $\mathrm{BF}$ was characterized using infrared testing and elemental analysis. To investigate the effect of $\mathrm{BF}-\mathrm{g}$-LA on the performance of PLA/BF/BF- $g$-LA composites, the phase morphology, thermal stability, and mechanical properties of the composites were characterized using scanning electron microscopy, thermogravimetric analysis, and universal material testing, respectively. The addition of BF- $g$-LA improved the interface compatibility between PLA and BF. When the BF- $g$-LA content was $2 \mathrm{phr}$, the tensile and impact strengths of PLA/BF/BF- $g$-LA composites were $55.3 \mathrm{MPa}$ and $9.56 \mathrm{~kJ} / \mathrm{m}^{2}$, representing $30 \%$ and $27 \%$ increases, respectively, relative to corresponding values for PLA/BF composites.
\end{abstract}

Keywords: bamboo flour; lactide; graft; polylactic acid; compatibility

\section{Introduction}

The heavy use and unreasonable disposal of plastics has exacerbated environmental pollution in recent years. Thus, the development of environmentally sustainable replacements for traditional plastics is becoming a hot topic of today's research and development [1,2]. Polylactic acid (PLA) is a biodegradable, biological base material made from renewable resources. Similar to traditional plastics, PLA can be processed with thermoplastic methods, and it has excellent physical properties such as high transparency, biocompatibility, and biodegradability; however, it is expensive to produce and it is not heat-resistant, which limits its rapid development [3-5]. Many researchers have investigated ways to reduce the cost of PLA production. For example, composites have been prepared by blending PLA with starch, wood fiber, bamboo fiber, cotton, linen, and other natural polymers or inorganic fillers. Lv et al. [6], Chuayjuljit et al. [7] and Liu et al. [8,9] blended wood powder with PLA, and Sajna et al. [10] blended banana fiber with PLA to prepare composite materials. Nezamzadeh et al. [11] prepared thermoplastic/starch composites (PLA/TPS) by melt blending PLA. Gunti et al. [12,13] prepared natural fiber reinforced biodegradable material with short jute and big elephant grass fibers blended with PLA. Jiang et al. [14] and Prasad et al. [15] investigated the mechanical and thermal properties of fiber reinforced PLA composites by treating the surface of sisal fibers with alkali and silica aerogels (SAG). Arjmandi et al. [16] enhanced PLA by blending it with cellulose nanowhiskers (CNWs) and montmorillonite (MMT), and then investigated the mechanical properties of the composites. Gu et al. [17] and Almeida et al. [18] observed the morphological and rheological 
behavior of PLA $/ \mathrm{CaCO}_{3}$ composites, and Picard et al. [19] investigated the crystallinity and barrier properties of montmorillonite/PLA composites.

Bamboo flour (BF) is a renewable, low-cost, natural polymer material; therefore, it is often blended with plastic to prepare bamboo/plastic composite materials. Wang et al. [20] grafted GMA onto PLA to synthesize PLA-g-GMA using benzoyl peroxide (BPO) and tert-butyl peroxy benzoate (TBPB) as initiators, and the PLA-g-GMA was used as a compatibilizer for PLA/BF composites. It was determined that PLA-g-GMA improved the interface compatibility between PLA and BF. Kang et al. [21] prepared PLA/BF composites by blending BF and PLA with a silane coupling agent, maleic anhydride, and acrylic acid. Wang et al. [22] treated bamboo flour with a sodium silicate solution, and then blended it with PVC to prepare PVC/BF composites. Lu et al. [23] and Lee [24] prepared PLA/BF and PP/BF composites by blending BF with PLA and PP, respectively, after alkali treatment. $\mathrm{Xu}$ et al. [25] and Ochi [26] prepared PBS/acetylated-BF composites by blending polybutylene succinate (PBS) with acetylated bamboo fiber. Gupta [27] prepared epoxy resin/BF composites using bamboo fiber reinforced with epoxy resin.

In this study, BF-g-LA was synthesized by grafting BF onto LA in the molten state using stannous octoate as a catalyst. Using BF-g-LA as a compatibilizer, we prepared PLA/BF/BF-g-LA composites by blending polylactic acid (PLA) with BF (PLA/BF, 85/15 wt \%) and investigated the effect of BF- $g$-LA on the performance of the composites. The use of BF- $g$-LA as a compatibilizer for PLA and BF melt blending has not been reported previously.

\section{Experimental Procedure}

\subsection{Materials and Reagents}

The following materials and reagents were used in this study: PLA (4032D, density: $1.24 \mathrm{~g} / \mathrm{cm}^{3}$, melt index: $7 \mathrm{~g} / 10 \mathrm{~min}\left(190{ }^{\circ} \mathrm{C}, 2.16 \mathrm{Kg}\right)$, NatureWorks company (Minnetonka, MN, USA)); BF (80-120 mesh, Mujiang Weihua Spice Factory, Jiangmen, China); stannous octoate (pure grade, Aladdin Chemistry Co. Ltd., Shanghai, China); toluene (pure grade, Beijing Chemical Factory, Beijing, China); chloroform (pure grade, Beijing Chemical Factory, Beijing, China); LA (pure grade, Plac Company Shanghai Representative Office, Shanghai, China); and antioxidant (YD-1010, Beijing Dilong Chemical Co. Ltd., Beijing, China).

\subsection{Principal Equipment Used}

\subsubsection{Grafting Reaction}

The following laboratory equipment was used in this procedure: a collective thermostatic heating magnetic stirrer (DF-101S, Bangxi Instrument Technology Co. Ltd., Shanghai, China); a horizontal constant speed electric stirrer (HD2004W, Shanghai Secretary Music Instrument Co. Ltd., Shanghai, China); a circulating aquatic multi-purpose vacuum pump (SHB-2IIIA, Linhai Yonghao Vacuum Equipment Co. Ltd., Zhejiang, China); a three-necked flask (1000 mL, Sichuan Shu Glass (Group) Co. Ltd., Sichuan, China); a beaker (500mL, Sichuan Shubo (Group) Co. Ltd., Sichuan, China); and glass rods (6 mm $\times 250 \mathrm{~mm}$, Beijing Humanities Huatai Biotechnology Co. Ltd., Beijing, China).

\subsubsection{Blending}

The following laboratory equipment was used in this procedure: an XSS-300 Torque Rheometer (E85-582, Shanghai Branch Chong Rubber Machinery Equipment Co. Ltd., Shanghai, China); a flat press (LP-S-50, Sweden LAB TECH, Boston, MA, USA); a microcomputer-controlled electronic universal testing machine, UTM (Chengde Jin Jian Detection Instrument Co. Ltd., Hebei, China); a cantilever beam impact testing machine, (XJUD-55, Chengde Jin Jian Detection Instrument Co. Ltd., Hebei, China); a thermogravimetric (TG)tester (Q5000IR, TA Co., New Castle, DE, USA); an infrared spectrometer (Nicolet iZ10, Thermo Scientific Co., Waltham, MA, USA); an elemental analyzer (vario EL, Elementar, 
Shanghai, China); a scanning electron microscope (TESCAN VEGA II, TESCAN s.r.o, Brno, Czech); a vacuum oven (DZG-6050, Shanghai Senxin Experimental Instrument Co. Ltd., Shanghai, China); and an electric air-blowing dryer (A101-E3, Shanghai Second Hardware Factory, Shanghai, China).

\subsection{Sample Preparation}

\subsubsection{Preparation of BF- $g$-LA}

First, BF was dried in a vacuum oven at $80{ }^{\circ} \mathrm{C}$ for $12 \mathrm{~h}$, and the appropriate amount of LA was dried in a vacuum oven at $40^{\circ} \mathrm{C}$ for over $12 \mathrm{~h}$.

The dried BF and LA were poured into a three-necked flask, and the grafting reaction was initiated. The reaction device is shown in Figure 1, and the specific material mass ratio, reaction temperature, stirring speed, and reaction time are listed in Table 1.

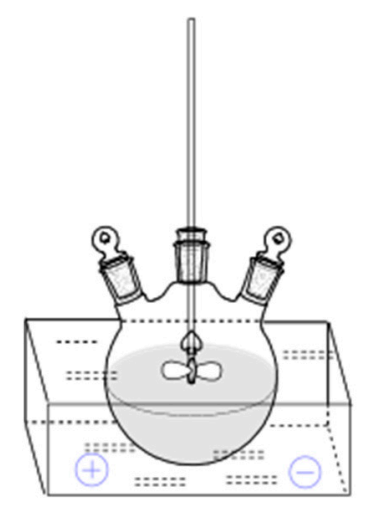

Figure 1. Experimental reaction device.

Table 1. Experimental reaction conditions.

\begin{tabular}{|c|c|c|c|c|c|c|c|c|c|c|}
\hline \multirow{2}{*}{$\begin{array}{l}\text { Grafting } \\
\text { Product } \\
\text { Abbreviation }\end{array}$} & \multirow{2}{*}{$\begin{array}{l}\text { BF/LAMmass } \\
\text { Ratio } \\
\left(\mathbf{W}_{\mathrm{BF}} / \mathrm{W}_{\mathrm{LA}}\right)\end{array}$} & \multicolumn{2}{|c|}{$\begin{array}{l}\text { Reaction } \\
\left(\mathrm{T} /{ }^{\circ} \mathrm{C}\right)\end{array}$} & Temperature & \multicolumn{2}{|c|}{$\begin{array}{l}\text { Stirring } \\
\left(\mathrm{v} / \mathrm{r} \cdot \min ^{-1}\right)\end{array}$} & \multirow{2}{*}{$\begin{array}{c}\text { Speed } \\
v_{\text {reaction }}\end{array}$} & \multicolumn{2}{|c|}{$\begin{array}{l}\text { Reaction } \\
(\mathrm{t} / \mathrm{h})\end{array}$} & \multirow{2}{*}{$\begin{array}{c}\text { Time } \\
t_{\text {reaction }}\end{array}$} \\
\hline & & $T_{\text {Melt }}$ & $T_{\text {swell }}$ & $T_{\text {reaction }}$ & $v_{\text {melt }}$ & $v_{\text {swell }}$ & & $t_{\text {melt }}$ & $t_{\text {swelling }}$ & \\
\hline $\mathrm{BF}-g-\mathrm{LA}_{1: 5}$ & $1: 5$ & 100 & 100 & 110 & 150 & 180 & 220 & 1 & 5 & 6 \\
\hline BF- $g$-LA $1: 6$ & $1: 6$ & 100 & 100 & 110 & 150 & 180 & 220 & 1 & 5 & 6 \\
\hline BF-g-LA $1: 7$ & $1: 7$ & 100 & 100 & 110 & 150 & 180 & 220 & 1 & 5 & 6 \\
\hline BF- $g$-LA $1: 8$ & $1: 8$ & 100 & 100 & 110 & 150 & 180 & 220 & 1 & 5 & 6 \\
\hline
\end{tabular}

Annotations: $T_{\text {Melt }}$, LA melting temperature; $T_{\text {swell }}, \mathrm{BF}$ swell temperature; $T_{\text {reaction, }}$ LA-BF reaction temperature; $v_{\text {Melt }}$, LA agitation speed when melted; $v_{\text {swell }}$, BF agitation speed when swelled; $v_{\text {reaction }}$ LA-BF reaction stirring speed; $t_{\text {melt }}$, time of LA melting; $t_{\text {swelling }}$, time of BF swelling; $t_{\text {reaction, }}$ LA-BF reaction time.

The experimental steps for the BF-LA grafting reaction are as follows:

(1) LA melting stage: The LA was weighed and placed into a three-necked flask. An oil bath was gradually warmed to $100{ }^{\circ} \mathrm{C}$, the stirrer was initiated, and the agitator gradually accelerated from a low speed to $150 \mathrm{r} / \mathrm{min}$ until all of the LA had melted (within $1 \mathrm{~h}$ ).

(2) BF swelling stage: BF was added to the three-necked flask, and the contents were mixed evenly at a stirring speed of $180 \mathrm{r} / \mathrm{min}$ and a temperature of $100{ }^{\circ} \mathrm{C}$ for $5 \mathrm{~h}$. During this process, $\mathrm{BF}$ was sufficiently swollen in the molten state of LA.

(3) LA and BF reaction phase: A distilled and dried toluene solution containing $2 \%$ stannous octoate (weight of LA) was added to the LA/BF solution. At the same time, the stirring speed was increased to $220 \mathrm{r} / \mathrm{min}$ and the temperature was elevated to $110^{\circ} \mathrm{C}$, initiating the chemical reaction between LA and BF. When the viscosity of the solution began to increase rapidly (about $1.5 \mathrm{~h}$ after 
the start of the reaction), stirring was stopped and the reaction proceeded for $6 \mathrm{~h}$, after which heating was stopped.

(4) Washing the reaction product: After the reaction product cooled, a chloroform solution was added to the three-necked flask and heated to $60^{\circ} \mathrm{C}$ from a low temperature. After the reaction product was completely dissolved, it was centrifuged. The above procedure was repeated four times to rinse off the untreated LA and free oligomers. The obtained BF- $g$-LA was dried in a vacuum at $60^{\circ} \mathrm{C}$ for $8 \mathrm{~h}$.

\subsubsection{Preparation of PLA/BF/BF- $g$-LA Composites}

BF was dried for $12 \mathrm{~h}$ in a vacuum oven at $80{ }^{\circ} \mathrm{C}$, and PLA was dried in a vacuum oven at $80^{\circ} \mathrm{C}$ for $8 \mathrm{~h}$.

The mixture formulation of the PLA/BF/BF- $g$-LA composite material is shown in Table 2.

Table 2. Mixture formulation (wt \%).

\begin{tabular}{cccc}
\hline PLA/wt $\%$ & BF/wt $\%$ & BF- $\boldsymbol{g}$-LA/phr & Antioxidant/phr \\
\hline 85 & 15 & 0 & 1 \\
85 & 15 & 2 & 1 \\
85 & 15 & 4 & 1 \\
85 & 15 & 6 & 1 \\
85 & 15 & 8 & 1 \\
\hline
\end{tabular}

According to Table 2, we weighed PLA and BF, mixed them evenly together, and transferred them to a mixer. The mixture was kneaded for $480 \mathrm{~s}$ at $180^{\circ} \mathrm{C}$ and $40 \mathrm{rpm}$. The blended materials were then pressed into a 4-mm-thick flat plate at $190^{\circ} \mathrm{C}$ by compression molding. The stretch spline conformed to GB/T1040-92 type I dumbbell specimens, and the impact spline conformed to GB/T1843-2008 non-gap specimens.

\subsection{Performance Testing and Characterization}

\subsubsection{Determination of BF-g-LA Content}

(1) Elemental analysis

A 3-mg sample of BF- $g$-LA was tested with an elemental analyzer (CHN mode). The temperature in the oxidation tube was $1150{ }^{\circ} \mathrm{C}$, and that in the reduction tube was $850^{\circ} \mathrm{C}$. Four sets of samples were tested, and the mean values were calculated.

(2) Infrared spectroscopy

The samples were blended with $\mathrm{KBr}$ powder and pressed into sheets. The sheets were scanned using an infrared spectrometer with a resolution of $4 \mathrm{~cm}^{-1}$ and a scanning frequency of 32 in the range of $400-4000 \mathrm{~cm}^{-1}$.

\subsubsection{Thermogravimetric Analysis of BF- $g$-LA Modified PLA/BF Composites}

The sample was subjected to thermogravimetric analysis under a nitrogen atmosphere $\left(40 \mathrm{mg} / \mathrm{min}\right.$ ) at a heating rate of $20^{\circ} \mathrm{C} / \mathrm{min}$. The determination range was $50-600{ }^{\circ} \mathrm{C}$.

\subsubsection{Testing of Mechanical Properties}

The samples were placed in a laboratory environment for $24 \mathrm{~h}$ and subjected to tensile testing according to GB/T 1040.2-2006 (IDT ISO 527.2-2: 1993) with a universal testing machine at a tensile speed of $2 \mathrm{~mm} / \mathrm{min}$.

Non-notched impact tests were performed using a cantilever beam impact testing machine (1J hammer) according to GB/T 1843-2008 (IDT ISO 180-2000). 


\subsubsection{Scanning Electron Microscopy}

The fracture surfaces of samples were sprayed with gold and observed at $2000 \times$ magnification with an acceleration voltage of $10 \mathrm{kV}$.

\section{Results and Discussion}

\subsection{Characterization of $B F-g-L A$}

\subsubsection{Infrared Spectroscopy}

The infrared spectra of BF-g-LA are shown in Figure 2, and the infrared absorption peaks for the main groups are listed in Table 3.

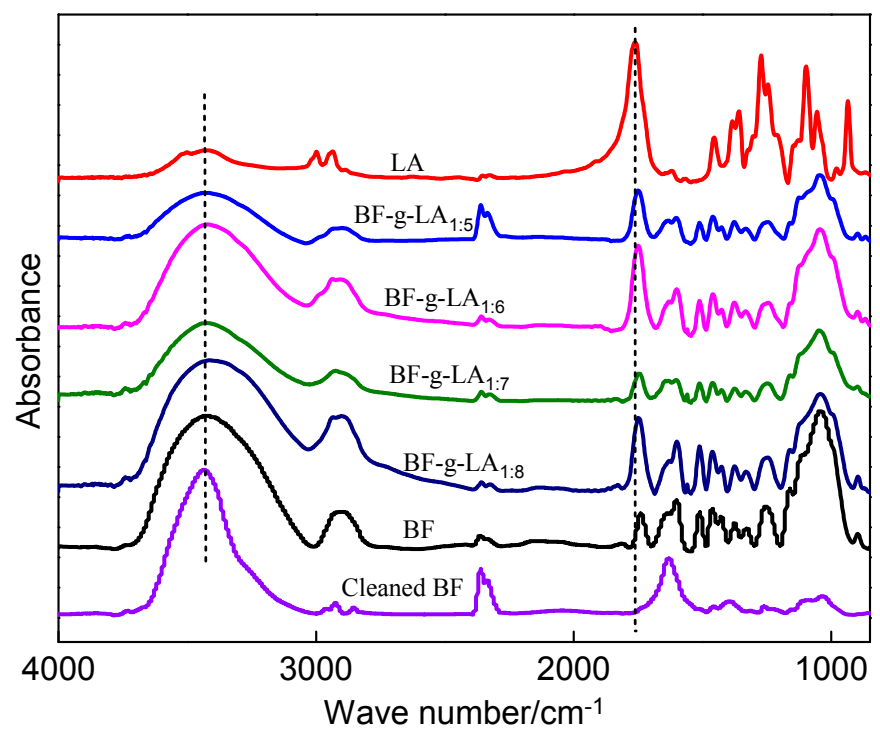

Figure 2. Infrared spectra of BF- $g-\mathrm{LA}$.

Table 3. Infrared absorption peaks of the main groups.

\begin{tabular}{cc}
\hline Wave Number/cm & \multicolumn{1}{c|}{ Group } \\
\hline 3500 & Hydroxyl O-Hstretching vibration \\
$1740-1762$ & Carbonyl C=O stretching vibration \\
1425,1460 & C-H bending, stretching vibration \\
1330 & Benzene ring stretching vibration \\
\hline
\end{tabular}

As shown in Figure 2 and Table 3, the carbonyl stretching vibration peak at 1740-1762 cm $\mathrm{cm}^{-1}$ disappeared after $\mathrm{BF}$ was rinsed with chloroform. The characteristic absorption peaks of the $\mathrm{C}-\mathrm{H}$ bond at 1425 and $1460 \mathrm{~cm}^{-1}$ (due to $\mathrm{C}-\mathrm{H}$ bending and stretching) and the benzene ring stretching vibration peak at $1330 \mathrm{~cm}^{-1}$ also disappeared. The disappearance of these peaks indicated that the structure of the BF was destroyed after rinsing with chloroform. However, these peaks still existed after BF was grafted onto LA, indicating that LA reacted with cellulose, hemicellulose, and lignin in the BF and the reaction product could not be rinsed with chloroform. In addition, the small molecular initiator, LA, free oligomer, and polymer were washed with chloroform to obtain high-purity BF-g-LA, so as to avoid holes formed by the small molecular initiator, LA, free oligomer cause poor compatibility between BF and PLA [28].

A model describing the reaction of the LA-BF grafting reaction is shown in Figure 3. 

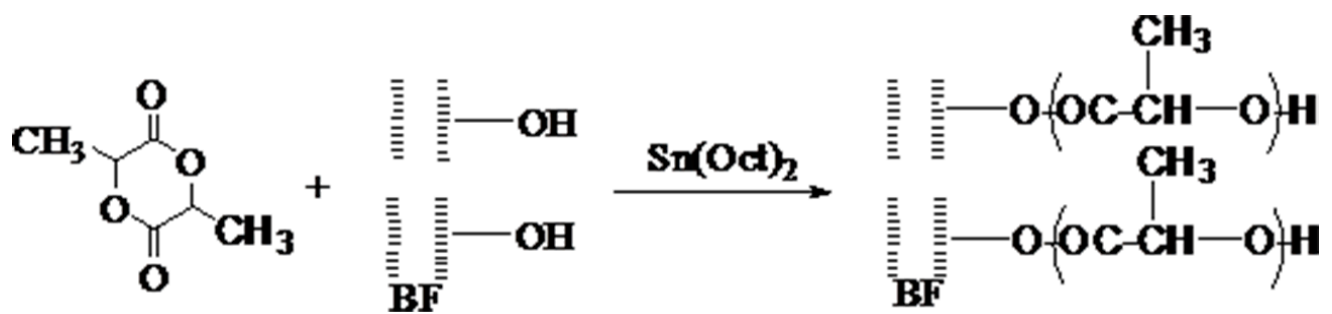

Figure 3. Reaction mechanism for Bamboo flour (BF) grafting lactide (LA).

The cell wall of BF fiber generally contains $40-50 \%$ cellulose, $20-30 \%$ hemicellulose, and $15-25 \%$ lignin in addition to small amounts of pectin, starch, pigment, inorganic materials, and other components. Cellulose is a linear homogeneous polymer formed by the polymerization of D-glucose via $\beta-1,4$-glycosidic bonds. Hemicellulose is a class of complex heterogeneous glycans consisting of pentose (xylose and arabinose), hexose (mannose, glucose, and galactose) and uronic acid. Lignin is a non-homogeneous amorphous polymer that contains three phenylpropane units (pineal alcohol, mustard alcohol, and coumarin).The benzene-propane units are linked by ether linkages (-O- and carbon-carbon bonds (-CC-).The hydroxyl groups in these components can be polymerized with lactide after the rings open, causing grafting of lactide onto BF.

The open ring of LA reacted with the hydroxyl groups on the surface of $\mathrm{BF}$, and the resulting molecular chain was longer in the BF- $g$-LA product (Figure 3); therefore, the product was no longer soluble in chloroform.

After washing with chloroform, the absorption peak of BF-g-LA was larger than that of BF, and the absorption peak of the carbonyl group at $1740 \mathrm{~cm}^{-1}$ shifted. The reason for these changes is that the stretching vibration peaks caused by carbonyl in BF-g-LA shifted between 1740 and $1762 \mathrm{~cm}^{-1}$ when LA was grafted onto the BF molecular chain [20].

The hydroxyl stretching vibration peak occurred at $3500 \mathrm{~cm}^{-1}$. The polarity of BF was strong owing to hydrogen bonding of the hydroxyl group. After BF was grafted, the molecular chain linked to the hydroxyl group lengthened and the polarity decreased. Subsequently, the strength of the hydrogen bonds decreased [29], and the intensity of the hydroxyl-induced stretching vibration peak was reduced.

\subsubsection{Elemental Analysis}

The carbon content in LA, BF, and BF-g-LA was determined using elemental analysis (Table 4).

Table 4. Carbon content of LA, BF, and BF- $g$-LA determined by elemental analysis.

\begin{tabular}{cccccccc}
\hline Concentration & LA & BF & $\begin{array}{c}\text { Cleaned BF by } \\
\text { Chloroform }\end{array}$ & BF- $g$-LA $1: 5$ & BF-g-LA 1:6 & BF- $g$-LA 1:7 & BF- $g$-LA $1: 8$ \\
\hline C wt $\%$ & $48.80 \pm 0.08$ & $49.83 \pm 0.26$ & $49.26 \pm 0.18$ & $48.86 \pm 0.09$ & $48.91 \pm 0.13$ & $48.97 \pm 0.06$ & $49.02 \pm 0.04$ \\
\hline
\end{tabular}

According to Table 4, the $\mathrm{C}$ content of pure BF, cleaned BF (with chloroform), and LA was $49.83 \%, 49.26 \%$, and $48.80 \%$, respectively. The $C$ content of the grafted products BF- $g$-LA $1: 5$, BF- $g$-LA $1: 6$, BF- $g$-LA $1: 7$, and BF- $g$-LA $1: 8$ was $48.86 \%, 48.91 \%, 48.97 \%$, and $49.02 \%$, respectively. These data indicate that LA was successfully grafted onto the BF molecular chain, which is consistent with results of the infrared spectroscopy analysis.

The content of LA in the grafted copolymer was calculated with Equation (1)

$$
w_{2}=\frac{x_{3}-x_{1} w_{1}}{x_{2}}
$$


where $x_{1}$ is the carbon content of $\mathrm{BF}(\mathrm{wt} \%) ; w_{1}$ is the mass fraction of $\mathrm{BF}(\mathrm{wt} \%) ; x_{2}$ is the carbon content of LA (wt \%); $w_{2}$ is the mass fraction of LA (wt \%); and $x_{3}$ is the carbon content of the graft product (wt \%) [20].

Based on the data in Table 4 and Equation (1), the content of LA in BF- $g$-LA I $_{1: 5}$, BF- $g$-LA $1: 6$, BF- $g$-LA $1: 7$, and BF- $g$-LA $1: 8$ was $94.17 \pm 8.7 \%, 89.32 \pm 12.6 \%, 83.50 \pm 6 \%$, and $78.64 \pm 7.3 \%$. It can be seen from the above analysis that the $\mathrm{BF}$ grafting rate was highest in $\mathrm{BF}-\mathrm{g}-\mathrm{LA}_{1: 5}$. Therefore, $\mathrm{BF}-\mathrm{g}-\mathrm{LA}_{1: 5}$ was used as a compatibilizer in subsequent experiments to study its compatibility in PLA and BF melt blending.

\subsection{Effect of BF-g-LA on the Thermal Properties of Composites}

The thermogravimetric curves (TG, DTG) of the BF- $g$-LA and PLA/BF/BF- $g$-LA composites are shown in Figure 4. The thermogravimetric temperature of BF-g-LA is shown in Table 5, and the thermogravimetric temperature of PLA/BF/BF-g-LA composites is shown in Table 6.

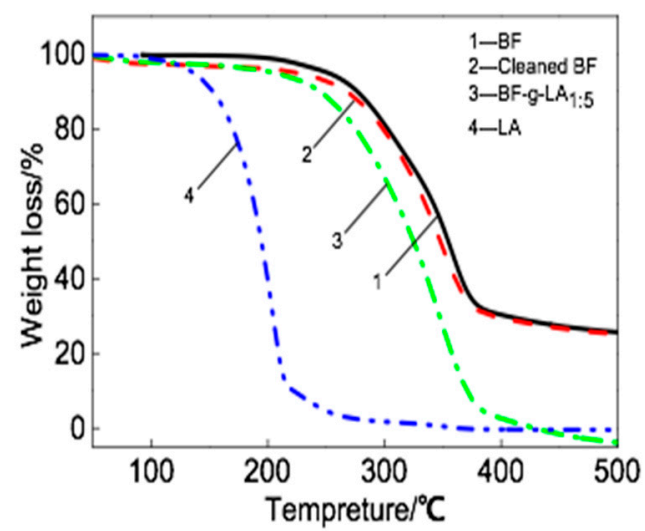

(a)

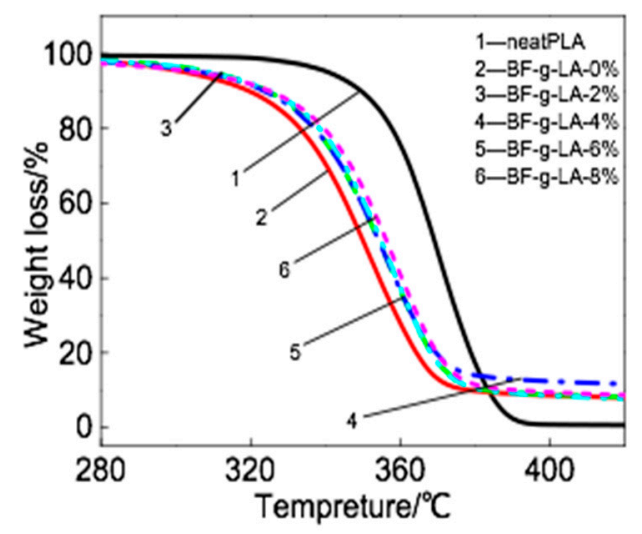

(c)

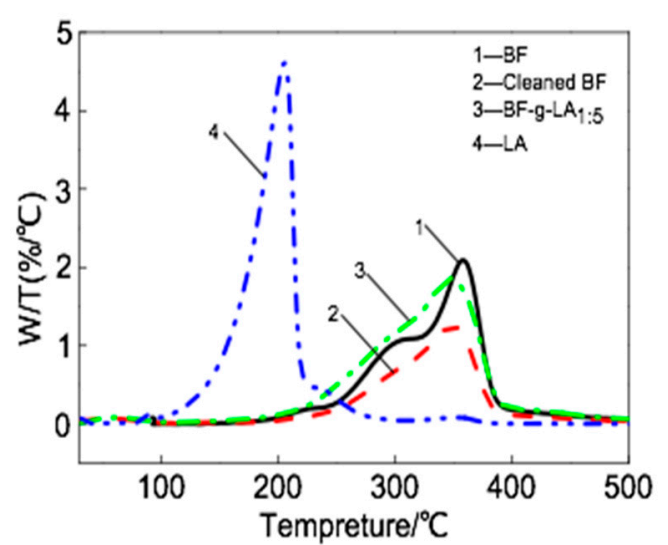

(b)

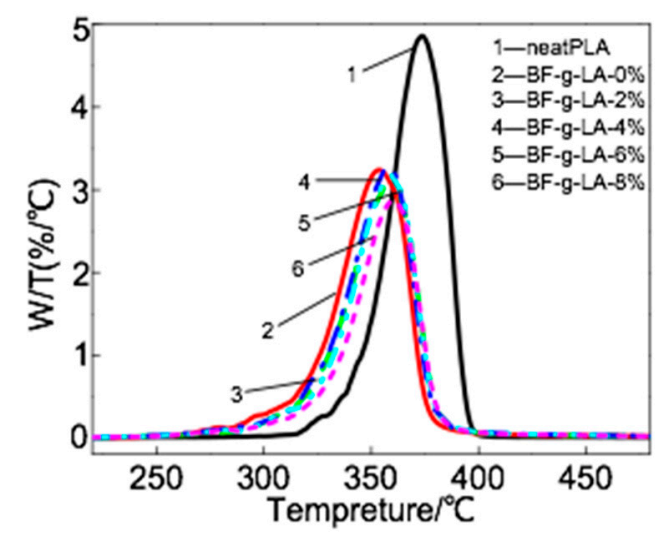

(d)

Figure 4. Thermal weightlessness curve for BF- $g$-LA and PLA/ BF/B- $g$-LA composites (a) TG curve for BF-g-LA; (b) DTG curve for BF-g-LA; (c) TG curves for PLA/BF/BF- $g$-LA composites; (d) DTG curve for PLA/BF/BF-g-LA composites.

Table 5. Thermogravimetric temperature of BF- $g$-LA.

\begin{tabular}{ccc}
\hline Samples & $\mathbf{5 \%}$ Weight-Loss Temperature $/{ }^{\circ} \mathbf{C}$ & DTG Peak Temperature $/{ }^{\circ} \mathbf{C}$ \\
\hline BF & 251 & 358 \\
Cleaned BF by chloroform & 224 & 349 \\
BF-g-LA $1: 5$ & 205 & 346 \\
LA & 136 & 205 \\
\hline
\end{tabular}


Table 6. Thermogravimetric temperature of PLA/BF/BF- $g$-LA composites.

\begin{tabular}{ccc}
\hline Samples & $\mathbf{5 \%}$ Weight-Loss Temperature $/{ }^{\circ} \mathbf{C}$ & DTG Peak Temperature $/{ }^{\circ} \mathbf{C}$ \\
\hline neat PLA & 341 & 373 \\
PLA/BF/BF- $g$-LA-0phr & 302 & 353 \\
PLA/BF/BF-g-LA-2phr & 313 & 362 \\
PLA/BF/BF-g-LA-4phr & 311 & 361 \\
PLA/BF/BF- $g$-LA-6phr & 312 & 362 \\
PLA/BF/BF- $g$-LA-8phr & 309 & 364 \\
\hline
\end{tabular}

As seen in Figure $4 \mathrm{a}, \mathrm{b}$ and Table 5, the $5 \%$ weight-loss temperature of $\mathrm{BF}$ was $251{ }^{\circ} \mathrm{C}$, and the maximum decomposition rate temperature was $358^{\circ} \mathrm{C}$. The $5 \%$ weight-loss temperature of LA was $136{ }^{\circ} \mathrm{C}$, and the maximum decomposition rate temperature was $205^{\circ} \mathrm{C}$. The $5 \%$ weight-loss and maximum decomposition rate temperatures of $\mathrm{BF}-\mathrm{g}-\mathrm{LA}_{1: 5}$ were between the corresponding values for $\mathrm{BF}$ and LA because the presence of LA in the grafted product reduced the thermal decomposition temperature of $\mathrm{BF}-\mathrm{g}-\mathrm{LA}_{1: 5}$. After washing with chloroform, hemicellulose and lignin in the BF were partly removed, and the weight loss temperature decreased.

The $5 \%$ weight-loss temperature of the PLA/BF composite without BF- $g$-LA was $302{ }^{\circ} \mathrm{C}$ and the maximum decomposition rate temperature was $353{ }^{\circ} \mathrm{C}$ (Figure $4 \mathrm{c}, \mathrm{d}$ and Table 6). After adding 2, 4, 6, and $8 \mathrm{phr}$ BF-g-LA, the 5\% weight-loss temperature of the composites was 313, 311, 312, and $309{ }^{\circ} \mathrm{C}$ and the maximum decomposition rate temperature was $362,361,362$, and $364{ }^{\circ} \mathrm{C}$, respectively. Thus, the $5 \%$ weight-loss temperature and the maximum decomposition rate temperature for PLA/BF/BF- $g$-LA composites improved after the addition of BF- $g$-LA. When two phr BF-g-LA was added, the $5 \%$ weight-loss temperature of the composite was $313{ }^{\circ} \mathrm{C}$ and the maximum decomposition rate temperaturewas $362{ }^{\circ} \mathrm{C}\left(9\right.$ and $11{ }^{\circ} \mathrm{C}$ higher than that of PLA/BF composites without BF- $g$-LA, respectively).

The above analysis shows that the addition of BF- $g$-LA improved the compatibility between PLA and $\mathrm{BF}$, and reinforced the thermal stability of PLA/BF composites.

\subsection{Effect of BF-g-LA on the Mechanical Properties of Composites}

The measured tensile and impact strengths of PLA/BF/BF- $g$-LA composites are shown in Figures 5 and 6, respectively.

The tensile strength of PLA/BF composites without BF- $g$-LA was $42.5 \mathrm{MPa}$, and the tensile strength of composites after addition of 2, 4, 6, and $8 \mathrm{phr}$ BF-g-LA was 55.3, 54.8, 52.7, and 55.4 MPa, respectively (Figure 5). When the content of BF- $g$-LA was 8 phr, the tensile strength of the composite was $55.4 \mathrm{MPa}$, which is $30 \%$ higher than that of unmodified composites.

The impact strength of PLA/BF composites without BF-g-LA was $7.5 \mathrm{~kJ} / \mathrm{m}^{2}$, and the impact strength of composites after addition of $2,4,6$, and $8 \mathrm{phr}$ BF- $g$-LA was $9.6 \mathrm{~kJ} / \mathrm{m}^{2}, 9.3 \mathrm{~kJ} / \mathrm{m}^{2}$, $8.7 \mathrm{~kJ} / \mathrm{m}^{2}$, and $8.5 \mathrm{~kJ} / \mathrm{m}^{2}$, respectively (Figure 6). The impact strength of composites reached a maximum value of $9.6 \mathrm{~kJ} / \mathrm{m}^{2}$ after addition of $2 \mathrm{phr} B F-g$-LA; this value was $27 \%$ higher than that of unmodified composites.

The tensile and impact strengths of PLA/BF composites were lower than corresponding values for pure PLA because the cellulose, hemicellulose, and lignin components of BF, contain large amounts of polar hydroxyl and phenolic hydroxyl groups (Figures 5 and 6). The interaction between BF and hydrophobic surface PLA interface was weak. Therefore, stress could not be transmitted effectively along the interface. As a result, the tensile and impact strengths of the composites were low and the mechanical properties of the composites decreased [30,31]. However, after adding BF-g-LA, the structure of LA in BF- $g$-LA improved the compatibility between BF and PLA, causing the tensile and impact strengths to increase. 


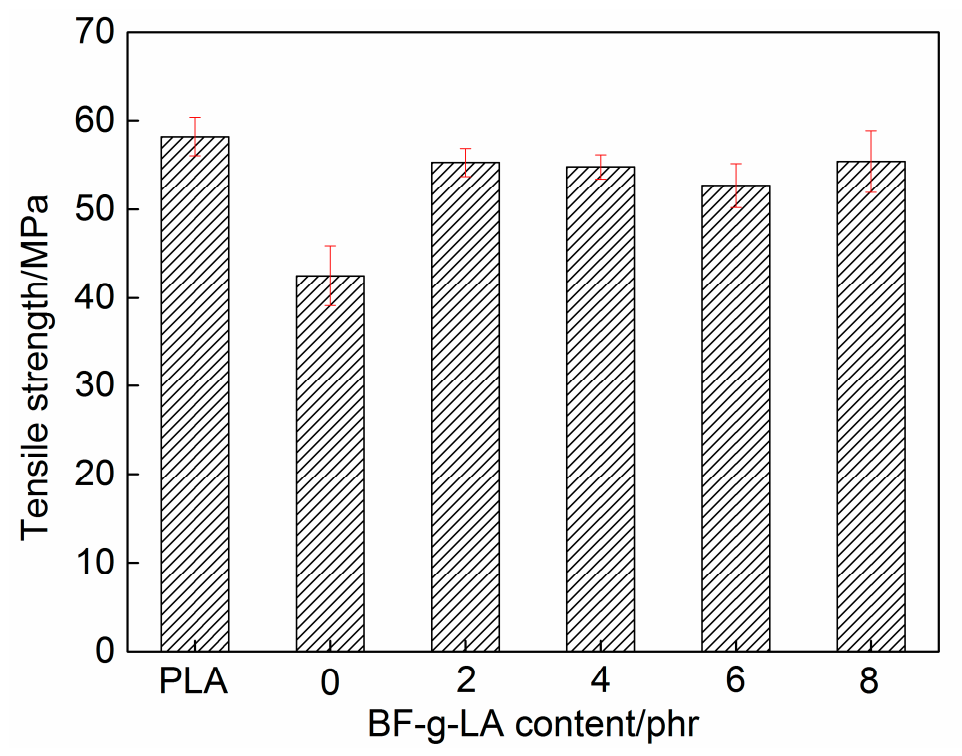

Figure 5. Tensile strength of PLA/BF/BF-g-LA composites.

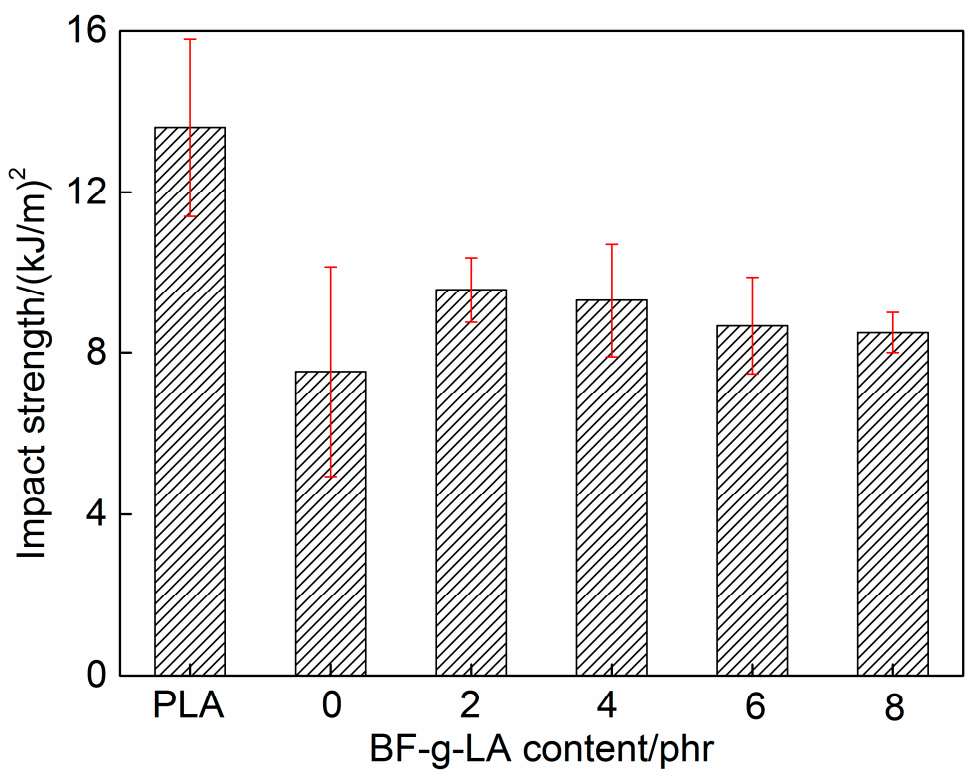

Figure 6. Impact strength of PLA/BF/BF-g-LA composites.

\subsection{Effect of BF-g-LA on the Morphology of the Fracture Surface of the Composites}

The morphologies of the impact fracture surfaces of PLA/BF/BF- $g$-LA composites are shown in Figure 7. The scanning electron microscopy image of the composite with no compatibilizer is shown in Figure 7a; composite images after the addition of BF-g-LA are shown in Figure 7b-e. 


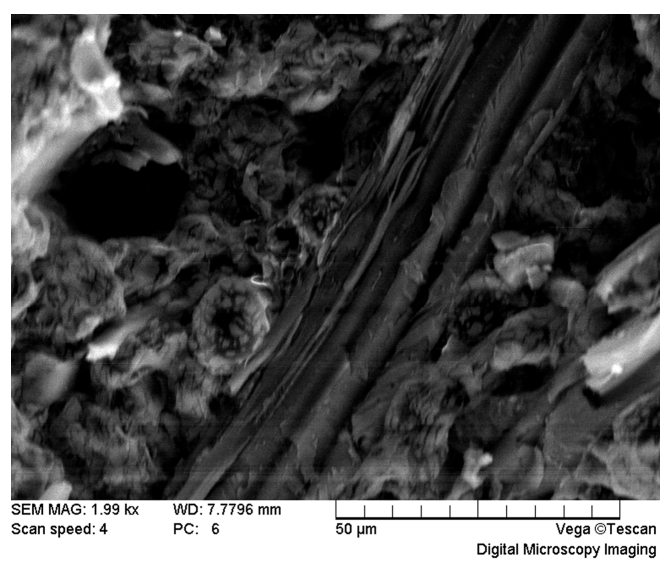

(a)

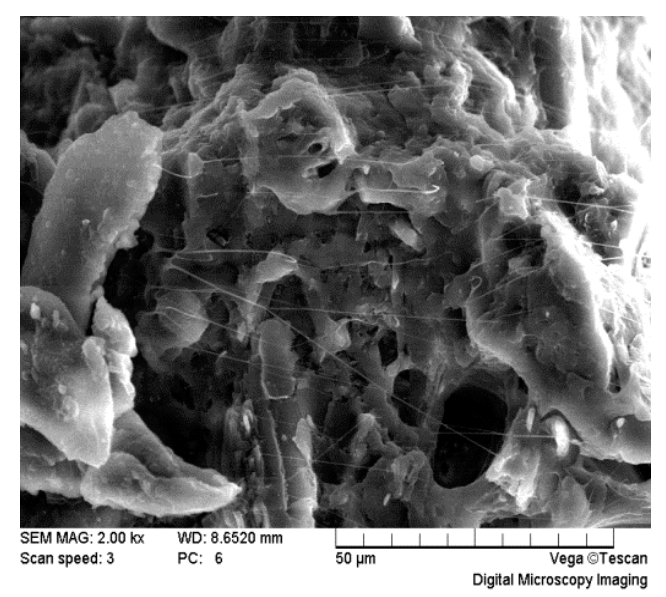

(c)

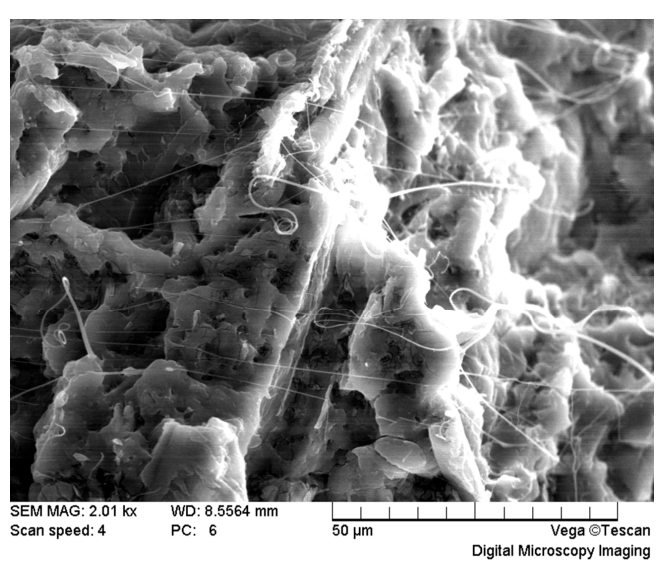

(b)

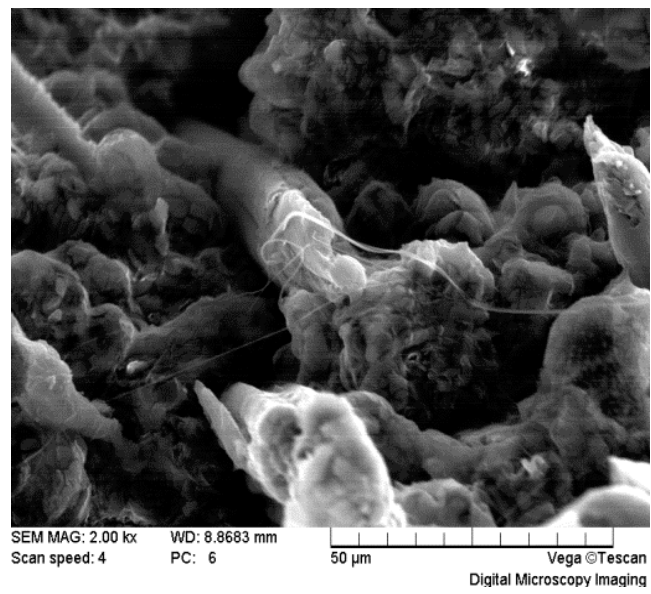

(d)

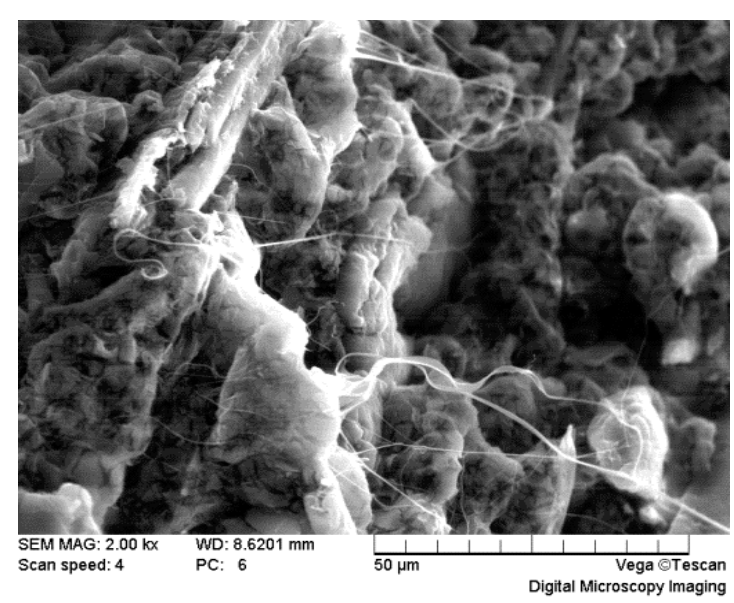

(e)

Figure 7. Scanning electron microscopy images $(\times 2000)$ of PLA/ BF/BF- $g$-LA impact surfaces (a) BF- $g$-LA, 0 phr; (b) BF- $g$-LA, 2 phr; (c) BF- $g$-LA, 4 phr; (d) BF- $g$-LA, 6 phr; (e) BF- $g$-LA, 8 phr.

As shown in Figure 7a, without the addition of BF-g-LA, a clear phase separation occurred between BF and PLA after melt blending, indicating poor compatibility of the unmodified PLA/BF $(85 / 15$ wt \%). 
The compatibility between BF and PLA improved after the addition of BF-g-LA, and the interface of the matrix was tightly connected (Figure $7 \mathrm{~b}-\mathrm{e}$ ). BF was dispersed and tightly enveloped in the PLA matrix. BF- $g$-LA acted as a reactive compatibilizer when the open ring in LA reacted with the hydroxyl group on the surface of $\mathrm{BF}$, thereby forming filamentous structures and enhancing the compatibility between BF and PLA. As a result, the two phases were closely connected, forming a relatively stable and strong interface layer. After the impact, BF first bore stress for separation, but because of the strong force between the two phases, the fiber was accompanied by the PLA matrix resin during separation, resulting in adhesion of the drawing phenomenon [32].

\section{Conclusions}

(1) Stannous octoate can initiate BF grafting onto LA to obtain BF- $g$-LA. The LA content in BF- $g$-LA $1: 5$, BF- $g$-LA $1: 6$, BF- $g$-LA 1:7, and BF- $g$-LA1 was $94.17 \%, 89.32 \%, 83.50 \%, 78.64 \%$, respectively.

(2) The addition of BF- $g$-LA during the preparation of PLA/BF/BF-g-LA composites resulted in the formation of a relatively strong interfacial layer. The tensile strength, impact strength, and thermal decomposition temperature increased. The addition of BF- $g$-LA effectively improved the compatibility between PLA and BF. The tensile and impact strengths of PLA/BF/BF-g-LA composites (addition of $2 \mathrm{phr}$ BF- $g$-LA) were $55.3 \mathrm{MPa}$ and $9.6 \mathrm{~kJ} / \mathrm{m}^{2}$, respectively, reflecting $30 \%$ and $27 \%$ increases compared with values for composites without BF-g-LA addition (42.5 MPa and $7.5 \mathrm{~kJ} / \mathrm{m}^{2}$, respectively)

Acknowledgments: The authors gratefully acknowledge the financial support of the National Science Found (Project No. 51473006, 51173005) and Science and Technology Innovation Team of BTBU for biobased green food packing (Project No. 0142132015).

Author Contributions: Yun-Xuan Weng conceived and designed the experiments; Xin-Yu Song and Meng Wang performed the experiments; Yun-Xuan Weng, Xin-Yu Song and Zhi-Gang Huang analyzed the data; Yun-Xuan Weng contributed reagents/materials/analysis tools; Xin-Yu Song wrote the paper.

Conflicts of Interest: The authors declare no conflict of interest.

\section{References}

1. Dorez, G.; Taguet, A.; Ferry, L.; Lopez-Cuesta, J.M. Thermal and fire behavior of natural fibers/PBS biocomposites. Polym. Degrad. Stabil. 2013, 98, 87-95. [CrossRef]

2. Zhou, Y.X.; Huang, Z.G.; Diao, X.Q.; Weng, Y.X.; Wang, Y.Z. Characterization of the effect of REC on the compatibility of PHBH and PLA. Polym. Test. 2015, 42, 7-25. [CrossRef]

3. Tsuji, H.; Sasaki, H.; Sato, H.; Gotoh, Y.; Ishikawa, J. Neuron attachment properties of carbon negative-ion implanted bioabsorbable polymer of poly-lactic acid. Nucl. Instrum. Methods Phys. Res. Sect. B 2002, 191, 815-819. [CrossRef]

4. Tarvainen, T.; Karjalainen, T.; Malin, M.; Pohjolainen, S.; Tuominen, J.; Seppala, J.; Jarvinen, K. Degradation of and drug release from a novel 2,2-bis(2-oxazoline) linked poly(lactic acid) polymer. J. Control. Release 2002, 81, 251-261. [CrossRef]

5. Gao, C.L.; Xia, Y.Z.; Ji, Q. Study on modification of polylactic acid. Mater. Rev. 2006, 20, 372-374.

6. Lv, S.; Gu, J.; Tan, H.; Zhang, Y. Modification of wood flour/PLA composites by reactive extrusion with maleic anhydride. J. Appl. Polym. Sci. 2016, 13. [CrossRef]

7. Chuayjuljit, S.; Wongwaiwattanakul, C.; Chaiwutthinan, P.; Prasassarakich, P. Biodegradable poly(lactic acid)/poly(butylene succinate)/wood flour composites: Physical and morphological properties. Polym. Compos. 2016. [CrossRef]

8. Liu, R.; Cao, J.; Peng, Y.; Chen, Y. Physical, mechanical, and thermal properties of micronized organo-montmorillonite suspension modified wood flour/poly(lactic acid) composites. Polym. Compos. 2015, 36, 731-738. [CrossRef]

9. Liu, R.; Peng, Y.; Cao, J. Thermal stability of organo-montmorillonite-modified wood flour/poly(lactic acid) composites. Polym. Compos. 2016, 37, 1971-1977. [CrossRef]

10. Sajna, V.P.; Mohanty, S.; Nayak, S.K. A study on thermal degradation kinetics and flammability properties of poly (lactic acid)/banana fiber/nanoclay hybrid bionanocomposites. Polym. Compos. 2015. [CrossRef] 
11. Ali Nezamzadeh, S.; Ahmadi, Z.; AfshariTaromi, F. From microstructure to mechanical properties of compatibilizedpolylactide/thermoplastic starch blends. J. Appl. Polym. Sci. 2017. [CrossRef]

12. Gunti, R.; Ratna Prasad, A.V.; Gupta, S. Preparation and properties of successive alkali treated completely biodegradable short jute fiber reinforced PLA composites. Polym. Compos. 2015, 37, 2160-2170. [CrossRef]

13. Gunti, R.; Ratna Prasad, A.V.; Gupta, S. Mechanical and degradation properties of natural fiber reinforced PLA composites: Jute, sisal, and elephant grass. Polym. Compos. 2016. [CrossRef]

14. Jiang, A.; Xu, X.; Wu, H. Preparation and properties of L-lactide-grafted sisal fiber-reinforced poly(lactic acid) composites. Polym. Compos. 2016, 37, 802-809. [CrossRef]

15. Prasad, C.V.; Sudhakara, P.; Prabhakar, M.N.; Shah, A.U.R.; Song, J.I. An Investigation on the Effect of Silica Aerogel Content on Thermal and Mechanical Properties of Sisal/PLA Nano Composites. Polym. Compos. 2016. [CrossRef]

16. Arjmandi, R.; Hassan, A.; Eichhorn, S.J.; Haafiz, M.K.M.; Zakaria, Z.; Tanjung, F.A. Enhanced ductility and tensile properties of hybrid montmorillonite/cellulose nanowhiskers reinforced polylactic acid nanocomposites. J. Mater. Sci. 2015, 50, 3118-3130. [CrossRef]

17. Gu, S.Y.; Zou, C.Y.; Zhou, K.; Ren, J. Structure-rheology responses of polylactide/calcium carbonate composites. J. Appl. Polym. Sci. 2009, 114, 1648-1655. [CrossRef]

18. De Almeida, J.F.M.; da Silva, A.L.N.; Escócio, V.A.; da Silva, A.H.M.D.F.T.; de Sousa, A.M.F.; Nascimento, C.R.; Bertolino, L.C. Rheological, mechanical and morphological behavior of polylactide/nano-sized calcium carbonate composites. Polym. Bull. 2016, 73, 1-15. [CrossRef]

19. Picard, E.; Espuched, E.; Fulchiron, R. Effect of an organo-modified montmorillonite on PLA crystallization and gas barrier properties. Appl. Clay Sci. 2011, 53, 58-65. [CrossRef]

20. Wang, Y.N.; Weng, Y.X.; Wang, L. Characterization of interfacial compatibility of polylacticacid and bamboo flour (PLA/BF) in biocomposites. Polym. Test. 2014, 36, 119-125. [CrossRef]

21. Kang, J.T.; Park, S.H.; Kim, S.H. Improvement in the adhesion of bamboo fiber reinforced polylactidecomposites. J. Compos. Mater. 2014, 48, 2567-2577. [CrossRef]

22. Wang, H.; Sheng, K.C.; Chen, J.; Mao, H.L.; Qian, X.Q. Mechanical and thermal properties of sodium silicate treated moso bamboo particles reinforced PVC composites. Sci. China Technol. Sci. 2010, 53, 2932-2935. [CrossRef]

23. Lu, T.; Liu, S.; Jiang, M.; Xu, X.; Wang, Y.; Wang, Z.Y.; Gou, J.; Hui, D.; Zhou, Z.W. Effects of modifications of bamboo cellulose fibers on the improved mechanical properties of cellulose reinforced poly(lactic acid) composites. Compos. Part B Eng. 2014, 62, 191-197. [CrossRef]

24. Lee, B.H.; Lee, J.W.; Lee, K.W.; Kim, C.W.; Kim, K.S.; Kim, Y.C. Effects of Ethylene-Octene Copolymer and Alkali Treatment of Bamboo Fiber (BF) on the Physical Properties of PP/BF Composites. Polymer 2016, 40, 607-613. [CrossRef]

25. Xu, X.L.; Zhang, M.; Qiang, Q.; Song, J.Q.; He, W.Q. Study on the performance of the acetylated bamboo fiber/PBS composites by molecular dynamics simulation. J. Compos. Mater. 2015, 50, 995-1003. [CrossRef]

26. Ochi, S. Flexural Properties of Long Bamboo Fiber/ PLA Composites. Open J. Compos. Mater. 2015, 5, 70-78.

27. Gupta, A. Synthesis, chemical resistance, and water absorption of bamboo fiber reinforced epoxy composites. Polym. Compos. 2016, 37, 141-145. [CrossRef]

28. Li, J.; Lu, X.L.; Zheng, Y.F. Effect of surface modified hydroxyapatite on the tensile property improvement of HA/PLA composite. Appl. Surf. Sci. 2008, 255, 494-497. [CrossRef]

29. Shen, C. Determination of Vapor-Liquid Equilibrium and Thermodynamics Model of Methanol-Ethanol-Water-Ionic Liquid System; Beijing University of Chemical Technology: Beijing, China, 2011.

30. Gong, X.; Xi, J.K.; Wu, H.W. Mechanical properties of sisal fiber reinforced polylactic acid biodegradable composites. Plastics 2010, 3, 26-29.

31. Li, X.G.; Wu, Y.Q.; Zheng, X.; Qin, H.Y. Advances in Research on Compatibility of Plant Fibers with Biodegradable Plastics. Plast. Technol. 2009, 37, 86-89.

32. Zhou, X.X.; Huang, S.S.; Chen, L.H.; Lin, Q.J. Water Absorbency and Surface Wettability of Polypropylene Composites Reinforced with Bamboo Powder. J. Fujian Agric. For. Univ. Nat. Sci. Ed. 2012, 41, 208-212. 